\title{
Direct multivariable controller tuning for internal combustion engine test benches
}

\author{
Thomas E. Passenbrunner ${ }^{\mathrm{a}, *}$, Simone Formentin ${ }^{\mathrm{b}}$, Sergio M. Savaresi ${ }^{\mathrm{c}}$, Luigi del $\operatorname{Re}^{\mathrm{a}}$ \\ a Institute for Design and Control of Mechatronical Systems, Johannes Kepler University Linz, 4040 Linz, Austria \\ b Dipartimento di Ingegneria, Università degli Studi di Bergamo, via Marconi 5, 24044 Dalmine (BG), Italy \\ ${ }^{\mathrm{c}}$ Dipartimento di Elettronica e Informazione, Politecnico di Milano, Piazza Leonardo da Vinci 32, 20133 Milano, Italy
}

Received 31 October 2013

Accepted 20 April 2014

Available online 21 May 2014

\section{Introduction}

The speed of a vehicle is the result of the engine torque - set by the driver via the accelerator pedal - and the load as a direct consequence of the road and vehicle conditions. Test benches are today often used to simulate the operation of an internal combustion engine in a vehicle. Both the torque and the speed at the crankshaft of the internal combustion engine need to track the values that the internal combustion engine would experience in the vehicle. The load torque has to be computed and enforced by the dynamometer at a test bench. If the torque profiles delivered by the dynamometer are satisfactory, measurements in a vehicle may be replaced by measurements at a test bench with significant advantages in terms of reproducibility, reduced time and costs when calibrating maps of an engine control unit or developing new engine control concepts.

The accuracy of the control significantly affects the validity of the measurements at a test bench. Therefore, the subject has received attention in different ways. A digital controller for a turbocharged Diesel engine as well as a direct current dynamometer is developed in Tuken, Fullmer, and VanGerpen (1990) using a closed-loop pole assignment technique to test an internal combustion engine with transient test cycles. The model reference adaptive control approach using Lyapunov stability theory to derive the parameters update law is applied to engine speed and torque control in Yanakiev (1998). Multivariable controls of the enginedynamometer system have become increasingly popular in recent times. The closed-loop reference tracking is maximized and excessive loop interactions are avoided in Bunker, Franchek, and Thomason (1997) by balancing the bandwidths of the loop transfer functions. In Gruenbacher and del Re (2008) a robust inverse tracking method is applied to control an internal combustion engine test bench achieving a high tracking performance. The inverse optimal control problem is solved in Gruenbacher, Colaneri, and del Re (2008), and in Passenbrunner, Sassano, and del Re (2011) an approximation of the solution of the optimization problem is calculated for an internal combustion engine test bench.

However, all the approaches mentioned above are based on a mathematical model of the system which consists of the test bench mechanics and the dynamometer, but also of the engine system under test. As dynamical test benches are mostly used for the development of engine systems, their behavior will change frequently due to different calibrations or to the exchange of components. Against this background, the derivation of new

\footnotetext{
* Corresponding author. Fax: +43732 24686213.

E-mail address: thomas.passenbrunner@jku.at (T.E. Passenbrunner).
} 
models can be quite time-consuming and is frequently omitted, under the assumption that the changes will not affect too much the performance of the closed-loop system.

Looking for a more efficient solution, an alternative approach that skips the explicit model phase and directly tunes the parameters of the controller from data, following the recent ideas in data-driven controller tuning, see e.g. Bazanella (2012) and Formentin, Hirsch, Savaresi, and del Re (2012), is suggested. The strategy employed herein is based on the method introduced in Formentin, Savaresi, and del Re (2012) and Formentin and Savaresi (2011a) for dealing with multivariable coupled controller design using a collection of open-loop data, which has already been applied on another challenging engine application in Formentin, Hirsch, et al. (2012).

In order to cope with the practical requirements of this setup, in particular with the integral behavior between the torque and the speed, these methods must be extended to work also with some of the data collected during the closed-loop operation. This extension makes the procedure suitable also for unstable systems. Notice that such an approach is different from classical direct methods like Hjalmarsson, Gevers, Gunnarsson, and Lequin (1998), in that it is non-iterative, and from standard direct adaptive control, see e.g. Wellstead and Zarrop (1991), in that it is an off-line method which allows for a fixed parameterization of the controller.

Measurements for the identification of the controller parameters are recorded at an internal combustion engine test bench equipped with a production light duty $2.0 \ell$ Diesel engine. The results are compared with the well tuned industrial standard, i.e. two gain scheduled single-input single-output PI controllers. The scheduling of the controller parameters of the industrial standard is done on the basis of the operating point that is defined from the engine speed and the accelerator pedal position. An adaptation of the controller parameters, learning approaches or similar is not provided by the industrial standard, see PUMA Open - avl.com (2014) for more details.

Both controllers show a comparable performance in tracking the references for the shaft torque and the engine speed. Disturbances caused by the couplings in the system are suppressed better by the proposed approach. Furthermore, the proposed approach accounts for a reduced effort and time required for tuning of the controller with respect to the industrial standard. The tuning of the test bench controller gets significantly simplified. Note that a prelimin-ary version of this work has been presented in Passenbrunner, Formentin, Savaresi, and del Re (2012).

The paper is organized as follows: Section 2 presents the system and provides, for better understanding, a brief mathema-tical description of the main dynamics. Section 3 recalls the basics of direct multivariable control design and extends the method for the case where some of the data are collected during closed-loop operation. The experimental design of a multivariable controller for an internal combustion engine test bench and a comparison with the industrial state-of-the-art system are finally illustrated in Section 4 and discussed in Section 5 . The paper is concluded by some remarks in Section 6.

\section{Internal combustion engine test benches}

The typical setup of an internal combustion engine test bench is depicted in Fig. 1, see Gruenbacher and del Re (2008) for further details. The internal combustion engine under test is connected via a flexible shaft with the electric dynamometer. The accelerator pedal position $\alpha$ and the set value $T_{D, \text { set }}$ of the dynamometer torque provide the inputs of the test bench. The engine speed $\omega_{E}$, the dynamometer speed $\omega_{D}$, the dynamometer torque $T_{D}$ and

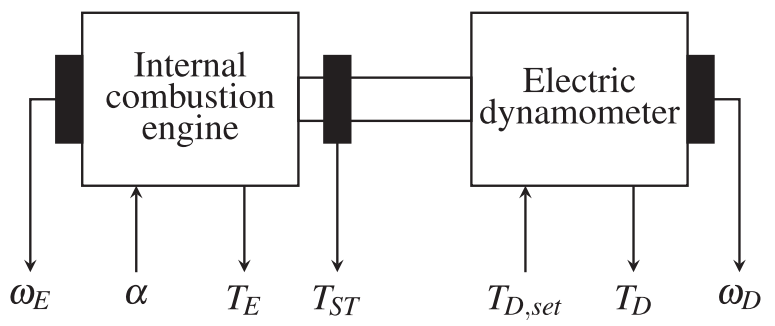

Fig. 1. Typical setup of an internal combustion engine test bench consisting of a dynamometer coupled to an internal combustion engine under test by a flexible shaft.

(sometimes) the shaft torque $T_{S T}$ are measured. In case the torque signals are not measured, the shaft torque $T_{S T}$ and the engine torque $T_{E}$ can be estimated from the available measurements, see Ortner, Gruenbacher, and del Re (2008) and Passenbrunner, Trogmann, and del Re (2012).

The entire mechanical part of the test bench can be modeled as a two-mass-oscillator as follows:

$$
\begin{aligned}
& \Delta \dot{\phi}=\omega_{E}-\omega_{D}, \\
& \dot{\omega}_{E}=\frac{1}{J_{E}}\left(T_{E}-T_{S T}\right), \\
& \dot{\omega}_{D}=\frac{1}{J_{D}}\left(T_{S T}-T_{D}\right)
\end{aligned}
$$

with the shaft torque

$T_{S T}=c \Delta \phi+d\left(\omega_{E}-\omega_{D}\right)$

and $\Delta \phi$ being the torsion angle of the connecting shaft. $J_{E}$ and $J_{D}$ are the inertia of the internal combustion engine and of the dynamometer, respectively. The inertia of the adapter flanges, the damping element, the shaft torque measurement device and the flywheel are already included in these values. The constant $c$ is the stiffness of the connecting shaft, $d$ the damping of the connecting shaft.

In general, modeling the behavior of the internal combustion engine is rather complicated. However, a simplified torque model of a light duty Diesel engine can be described by

$\dot{T}_{E}=-\tau\left(\omega_{E}, \alpha\right) T_{E}+\tau\left(\omega_{E}, \alpha\right) m\left(\omega_{E}, \alpha\right)$.

The varying parameter $\tau\left(\omega_{E}, \alpha\right)$ depends on the operating point defined by the accelerator pedal position $\alpha$ and the engine speed $\omega_{E}$, whereas $m\left(\omega_{E}, \alpha\right)$ represents a nonlinear static map with respect to $\alpha$ and $\omega_{E}$ which is bounded from below and above. For accelerator pedal position $\alpha$ close to $\alpha=0$, the drag torque of about $-30 \mathrm{~N}$ m appears. The maximum engine torque $T_{E}$ is limited to approximately $250 \mathrm{~N} \mathrm{~m}$. Furthermore, for actual internal combustion engines, the static engine torque also depends on the parameterization of the engine control unit.

The dynamics of the employed electric dynamometer can be neglected as it is much faster than those of the other components. Within the range of maximum torque and maximum rate of change, the torque of the dynamometer can then be described as

$T_{D}=T_{D, \text { set }}$

By exploiting the relationship $\Delta \omega=\omega_{E}-\omega_{D}$, the model (1)-(3) can be rewritten in its state-space representation as

$\dot{x}_{1}=x_{2}$,

$\dot{x}_{2}=-c \frac{J_{E}+J_{D}}{J_{E} J_{D}} x_{1}-d \frac{J_{E}+J_{D}}{J_{E} J_{D}} x_{2}+\frac{1}{J_{E}} x_{4}+\frac{1}{J_{D}} u_{2}$,

$\dot{x}_{3}=-\frac{c}{J_{E}} x_{1}-\frac{d}{J_{E}} x_{2}+\frac{1}{J_{E}} x_{4}$,

$\dot{x}_{4}=-\tau\left(x_{3}, u_{1}\right) T_{E}+\tau\left(x_{3}, u_{1}\right) m\left(x_{3}, u_{1}\right)$,

$y_{1}=x_{3}$, 
$y_{2}=c x_{1}+d x_{2}$

with the state vector

$\mathbf{x}=\left[\begin{array}{llll}x_{1} & x_{2} & x_{3} & x_{4}\end{array}\right]^{\top}=\left[\begin{array}{llll}\Delta \phi & \Delta \omega & \omega_{E} & T_{E}\end{array}\right]^{\top}$.

The input vector and the output vector are, respectively,

$\mathbf{u}=\left[\begin{array}{ll}u_{1} & u_{2}\end{array}\right]^{\top}=\left[\begin{array}{ll}\alpha & T_{D, s e t}\end{array}\right]^{\top}$

$\mathbf{y}=\left[\begin{array}{ll}y_{1} & y_{2}\end{array}\right]^{\top}=\left[\begin{array}{ll}\omega_{E} & T_{S T}\end{array}\right]^{\top}$

with the dimension $n_{u}=2$ of the input vector and the dimension $n_{v}=2$ of the output vector. The engine speed $\omega_{E}$ and the torque $T_{S T}$ at the crankshaft of the internal combustion engine must be tracked simultaneously to simulate the operation in a vehicle. In a decoupled control architecture, e.g. when testing a light duty engine, the torque at the crankshaft is usually regulated using the internal combustion engine, whereas the speed varies according to the dynamometer. However, such a scheme neglects the coupling effects of the two dynamics, and therefore may yield unacceptable performance in case of large excitation of torque and speed trajectories.

On the other hand, the design of a centralized control law is all but straightforward as the coupling effect is hard to be described accurately. Moreover, the stiffness and damping of the proposed model are sometimes uncertain and difficult to identify from the suited tests. This is another advantage of the proposed method, as a centralized controller can be designed without undertaking a full modeling study.

\section{Direct data-driven multivariable control system design}

\subsection{Model-reference control design from data}

Let the linear multivariable plant $\mathbf{G}\left(q^{-1}\right)$, where $q^{-1}$ denotes the backward shift operator, be the transfer function describing the linearization of the full test bench dynamics (4) in the neighborhood of a working point. Given a desired linear closedloop behavior $\mathbf{M}$, the objective of the proposed control problem could be reformulated as the problem of designing a multi-input multi-output (MIMO) controller $\mathbf{K}\left(q^{-1}, \boldsymbol{\rho}\right)$ of order $n$, parameterized through $\rho$, that minimizes (see Fig. 2)

$J_{M R}(\boldsymbol{\rho})=\left\|\mathbf{M}-(\mathbf{I}+\mathbf{G K}(\boldsymbol{\rho}))^{-1} \mathbf{G K}(\boldsymbol{\rho})\right\|_{2}^{2}$,

where $\mathbf{I}$ is the $\left(n_{y} \times n_{y}\right)$ identity matrix. Notice that (5) is nonconvex with respect to the controller parameters $\rho$.

Define the discrete-time control law as

$\mathbf{u}(t)=\mathbf{u}(t-1)+\sum_{k=0}^{n} \mathbf{B}_{k} \mathbf{e}(t-k)$,

where $\mathbf{B}_{k} \in \mathbb{R}^{n_{u} \times n_{y}}$ are matrices containing the unknown parameters such that

$\boldsymbol{\rho}=\left[\begin{array}{lll}\operatorname{vec}^{\top}\left(\mathbf{B}_{0}\right) & \ldots & \operatorname{vec}^{\top}\left(\mathbf{B}_{n}\right)\end{array}\right]^{\top}$

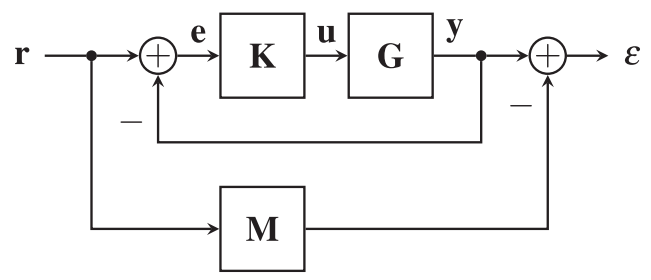

Fig. 2. Scheme of the model reference control problem with the linear multivariable plant $\mathbf{G}$, the desired linear closed-loop behavior $\mathbf{M}$ and the multi-input multi-output controller $\mathbf{K}$. with $\operatorname{vec}\left(\mathbf{B}_{k}\right)$ reshaping the matrix $\mathbf{B}_{k}$ to a row vector. The error is $\mathbf{e}(t)=\mathbf{r}(t)-\mathbf{y}(t)$ with $\mathbf{r}(t)$ being the reference signal. The regression form - that will be useful later on - of this controller is

$$
\begin{aligned}
\mathbf{u}(t) & =\mathbf{u}(t-1)+\mathbf{B}_{0} \mathbf{e}(t)+\cdots+\mathbf{B}_{n} \mathbf{e}(t-n) \\
& =\mathbf{u}(t-1)+\left[\mathbf{e}^{\top}(t) \otimes \mathbf{I} \ldots \mathbf{e}^{\top}(t-n) \otimes \mathbf{I} \boldsymbol{\rho}\right. \\
& =\mathbf{u}(t-1)+\boldsymbol{\varphi}^{\top}(t) \boldsymbol{\rho},
\end{aligned}
$$

where $\otimes$ denotes the Kronecker matrix product and the last equality defines $\varphi(t)$.

Consider now that a set of (persistently exciting, Ljung, 1998)

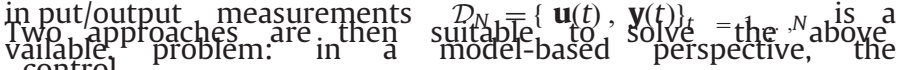
minimization of (5) can be achieved either by identifying a model of the plant from measured data or by linearizing the nonlinear system (4) and identifying its physical parameters (in a gray-box fashion). In both

cases, a model $\hat{\mathbf{G}}$ of the system is given, therefore, the modelreference control problem can be solved by minimizing $J_{M R}(\rho)$ evaluated in $\hat{\mathbf{G}}$. This approach would obviously be very sensitive to modeling errors and the closed-loop performance would be subsequently limited by the quality of the model. A different approach is the so-called "direct" data-driven philosophy that allows tuning directly the control action using data only, without first identifying a model of the plant. In this paper, the method in Formentin and Savaresi (2011a) will be employed. However, since in Formentin and Savaresi (2011a) only open-loop data can be used to guarantee a consistent estimate, the method will be herein reformulated and extended to be consistent using any data collection.

\subsection{A direct perspective}

The rationale behind the method refers to the Virtual Reference Feedback Tuning idea, fixed and extended in Campi, Lecchini, and Savaresi (2002) and Formentin and Savaresi (2011b), respectively, for linear time invariant systems and linear parameter varying scalar systems.

The main idea of the method is very simple. The basis observation is that, if input and output data are generated by the "ideal" closed-loop system, the closed-loop complementary sensitivity function would be, by definition, equal to $\mathbf{M}\left(q^{-1}\right)$. The reference signal feeding this "virtual loop", referred to as "virtual reference" signal from now on, could be then computed as (see Fig. 3) $\mathbf{r}_{v}(t)=\mathbf{M}^{-1}\left(q^{-1}\right) \mathbf{y}(t)$.

It follows that the corresponding "virtual error" signal is $\mathbf{e}_{v}(t)=\mathbf{r}_{v}(t)-\mathbf{y}(t)$.

The "ideal" controller is the one that generates $\mathbf{u}(t)$ when fed by $\mathbf{e}_{v}(t)$. Following this rationale, the control design issue turns out to be a simple identification problem, where the optimal controller is the one that best approximates the "ideal" one in the given class.

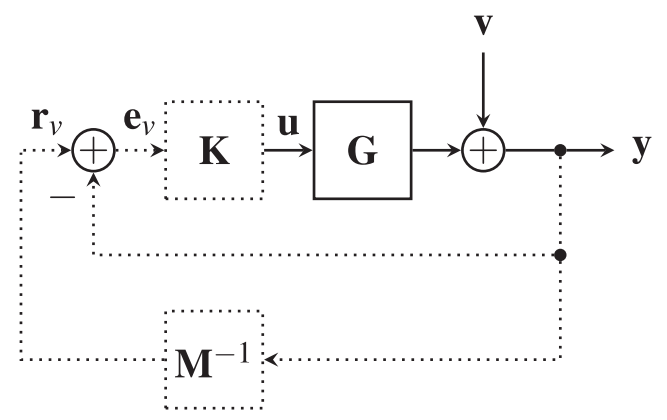

Fig. 3. Graphical view of the Virtual Reference Feedback Tuning rationale. 
Moreover, the optimization procedure is still convex and therefore the global minimum of (5) is guaranteed to be reached. The cost index to be carried out is, in the noiseless case,

$J_{V R}^{N}(\boldsymbol{\rho})=\frac{1}{N} \sum_{k=1}^{N}\left\|\mathbf{u}_{L}(k)-\mathbf{K}(\boldsymbol{\rho}) \mathbf{e}_{L}(k)\right\|_{2}^{2}$,

where $\mathbf{u}_{L}(t)=\mathbf{L} \mathbf{u}(t), \mathbf{e}_{L}(t)=\mathbf{L} \mathbf{e}_{v}(t)$ and $\mathbf{L}$ is a generic data prefilter. The prefilter $\mathbf{L}$ is needed to make (5) and (7) equal in the

neighborhood of the minimum point and to best shape the

bias in case of an unachievable reference model, see

Formentin and Savaresi (2011a).

Specifically, in Formentin and Savaresi (2011a), it has been illustrated that the best bias shaping is given by a filter that depends on the optimal controller. Then, the suboptimal selection $\mathbf{L}$ $=\mathbf{M}$ is instead proposed and implemented. This choice makes the asymptotic frequency-wise expression of $J_{V R}^{N}(\rho)$ equal to

$\tilde{J}_{V R}(\boldsymbol{\rho})=\frac{1}{2 \pi} \int_{-\pi}^{\pi} \operatorname{tr}[\mathbf{M}-\mathbf{K}(\boldsymbol{\rho}) \mathbf{G}(\mathbf{I}-\mathbf{M})] \Phi_{u u}[\mathbf{M}-\mathbf{K}(\boldsymbol{\rho}) \mathbf{G}(\mathbf{I}-\mathbf{M})]^{H} \mathrm{~d} \omega$,

that is also the frequency-wise version of the model-reference cost

$\tilde{J}_{M R}(\boldsymbol{\rho})=\left\|\left(\mathbf{M}-(\mathbf{I}+\mathbf{K}(\boldsymbol{\rho}) \mathbf{G})^{-1} \mathbf{K}(\boldsymbol{\rho}) \mathbf{G}\right) \Phi_{u u}^{1 / 2}\right\|_{2}^{2}$

with $\Phi_{u u}$ being the autocorrelation of the control input $\mathbf{u}$. Notice that (8) is analogous to the model-reference cost criterion, if the model to match is the input complementary sensitivity function and the actual sensitivity can be made close to the "ideal" one. In Formentin and Savaresi (2011a), simulations have shown that this setting guarantees very good performance even if the filter is suboptimal.

\subsection{The case of mixed open-/closed-loop noisy data}

Direct minimization of (7) would yield biased results using noisy data, i.e. if $\mathbf{v} \neq 0$ in Fig. 3. More specifically, it can be easily checked that the asymptotic frequency-wise expression of $J_{V R}^{N}(\rho)$ would become

$$
\begin{aligned}
\tilde{J}_{V R}(\boldsymbol{\rho})= & \tilde{J}_{M R}(\boldsymbol{\rho})+\frac{1}{2 \pi} \int_{-\pi}^{\pi} \operatorname{tr}[\mathbf{K}(\boldsymbol{\rho}) \mathbf{G}(\mathbf{I}-\mathbf{M})] \Phi_{v v}[\mathbf{K}(\boldsymbol{\rho}) \mathbf{G}(\mathbf{I}-\mathbf{M})]^{H} \mathrm{~d} \omega \\
& +\frac{1}{\pi} \int_{-\pi}^{\pi} \operatorname{tr}[\mathbf{K}(\boldsymbol{\rho}) \mathbf{G}(\mathbf{I}-\mathbf{M})] \Phi_{u v}[\mathbf{M}-\mathbf{K}(\boldsymbol{\rho}) \mathbf{G}(\mathbf{I}-\mathbf{M})]^{H} \mathrm{~d} \omega,
\end{aligned}
$$

thus generally leading to a wrong asymptotic estimate.

However, a decorrelation cost function can be introduced to make the estimate consistent, see Formentin and Savaresi (2011a) and Soderstrom and Stoica (1983). In this work, a different decorrelation cost function will be introduced, sharing the same global minimizer of (5) and (7) and guaranteeing the convergence to the optimal controller also in case of noisy input/output data. The difference with the method in Formentin and Savaresi (2011a) is that now data can be collected either in open-loop or in closedloop. Such a criterion is

$J_{d}^{N}(\boldsymbol{\rho})=(\mathbf{q}-\mathbf{Q} \boldsymbol{\rho})^{\top} \hat{\mathbf{W}}^{-1}(\mathbf{q}-\mathbf{Q} \boldsymbol{\rho})$,

where

$\mathbf{Q}=\frac{1}{N} \sum_{k=1}^{N} \zeta_{L}(k) \otimes \boldsymbol{\varphi}_{L}(k)$,

$\mathbf{q}=\frac{1}{N} \sum_{k=1}^{N} \boldsymbol{\zeta}_{\mathbf{L}}(k) \otimes \mathbf{u}_{L}(k)$

$\boldsymbol{\zeta}_{L}(t)=\mathbf{L} \boldsymbol{\zeta}(t)$,

$\boldsymbol{\varphi}_{L}(t)=\left[\mathbf{e}_{L}^{\top}(t) \otimes \mathbf{I} \ldots \mathbf{e}_{L}^{\top}(t-n) \otimes \mathbf{I}\right]^{\top}$,

and $\hat{\mathbf{W}}$ is a consistent estimate of the residual covariance matrix

$\mathbf{W}=\mathbb{E}\left[(\mathbf{q}-\mathbf{Q} \rho)(\mathbf{q}-\mathbf{Q} \rho)^{\top}\right]$. $\zeta(t)$ is an extended instrumental variable of the type defined in Stoica and Jansson (2002), that is

$\zeta(t)=\left[\begin{array}{c}\mathbf{w}(t+l) \\ \vdots \\ \mathbf{w}(t-l)\end{array}\right]$,

where

$\mathbf{w}(t)=\left[\begin{array}{llll}w_{1}(t) & \ldots & w_{n_{u}}(t)\end{array}\right]^{\top}$

is uncorrelated with $\mathbf{v}$ and $l$ is a sufficiently large integer. In a noiseless setting, if $l$ is large (at limit when it tends to infinity), $\boldsymbol{\rho}=\boldsymbol{\rho}_{o}$ asymptotically implies $\mathbf{Q} \boldsymbol{\rho}-\mathbf{q}=\mathbf{0}$.

The minima of ( 7 ) and (10) are the same and are given by

$\hat{\boldsymbol{\rho}}=\left(\mathbf{Q}^{\top} \hat{\mathbf{W}}^{-1} \mathbf{Q}\right)^{-1}\left(\mathbf{Q}^{\top} \hat{\mathbf{W}}^{-1} \mathbf{q}\right)$.

At the same time, $\mathbf{w}(t)$ and the noise signals are uncorrelated and the effect of the noise becomes negligible as $N \rightarrow \infty$, see Stoica and Jansson (2002) for further details. Moreover, the use of $\hat{\mathbf{w}}^{-1}$ allows us to shape the variance of the estimate and thus increase the statistical performance of the method.

In case of noisy data, the instrument $\zeta(t)$ is built so as to decorrelate the input and the noise regressors. More specifically,

if $\mathbf{w}$ is uncorrelated with $\mathbf{v}, \mathbf{Q}$ and $\mathbf{q}$ become asymptotically independent of the noise (the sum tends to the expected value, as $N$ grows) and $\mathbf{Q} \boldsymbol{\rho}-\mathbf{q}$ approaches zero. In Formentin and Savaresi (2011a), where only open-loop dataset are considered, $\mathbf{w}(t)=\mathbf{u}(t)$ can be safely selected, in order to guarantee consis-tency without performing new experiments. If the $i$-th output signal trajectory is collected during closed-loop operation, the corresponding term of $\mathbf{w}(t)$ cannot be chosen in the same way as $\mathbf{w}$ would be correlated with v. In this paper, the problem is solved by filling the instrumental variable with $w_{i}(t)=r_{i}(t)$, where $r_{i}$ is the reference trajectory of the $i$-th loop used in the experiment.

A comparison of the performance of a multivariable controller tuned with data recorded in open-loop and recorded in closedloop on a benchmark example (see Yeddanapudi \& Potvin, 1997) is given in Passenbrunner, Formentin, et al. (2012). This example clearly shows the advantages of using closed-loop data.

\section{Experimental results}

The method described in the previous section with the proposed modifications can now be used to design a multivariable controller for the application of interest in this paper. In particular, a test bench equipped with a production $2.0 \ell$ light duty Diesel engine is used to evaluate experimentally the performance, see Fig. 4. The internal combustion engine offers a power of about $130 \mathrm{~kW}$ and a maximum torque of $250 \mathrm{~N} \mathrm{~m}$, the maximum torque of the electric dynamometer is restricted to $300 \mathrm{~N} \mathrm{~m}$ by physical limitations.

In a first step, a persistently exciting experiment, see Ljung (1998), is performed to collect an input/output dataset using the mixed open-/closed-loop scheme depicted in Fig. 5. While the torque is set via feed-forward accelerator pedal position command $\alpha$, the engine speed $\omega_{E}$ needs to be feedback controlled due to the integral behavior of the test bench. In this specific case, a simple empirically tuned PI controller is used to set the desired torque $T_{D, \text { set }}$ of the dynamometer.

The collected input/output dataset is shown in Fig. 6. In the first graph the desired accelerator pedal position $\alpha$ is given. The second graph and the third graph show the dynamometer torque $T_{D}$ and the shaft torque $T_{S T}$, respectively. The fourth graph depicts both the reference $\omega_{E \text {,ref }}$ of the engine speed and the measured engine speed $\omega_{E}$ and the fifth graph shows the tracking error between the desired engine speed $\omega_{E, \text { ref }}$ and the measured engine speed $\omega_{E}$. 


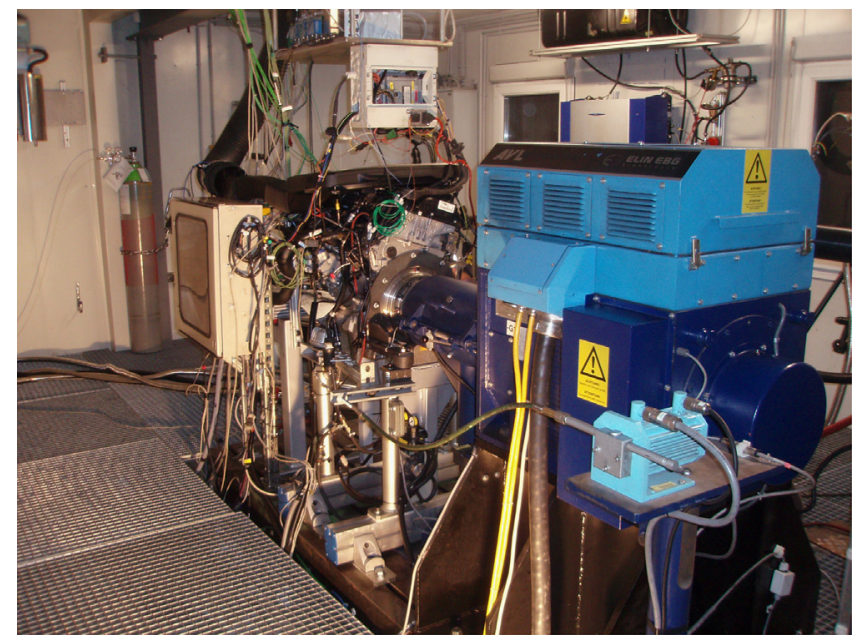

Fig. 4. Internal combustion engine test bench equipped with an electric dynamometer at Johannes Kepler University, used for the tests. The internal combustion engine with its ancillary units is shown at the left-hand side, the electric dynamometer at the right-hand side.

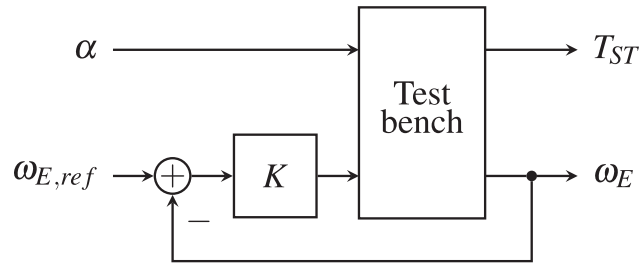

Fig. 5. Internal combustion engine test bench and a simple speed controller used to record measurement data for controller design.

Note that the experiment in Fig. 6 only visits a limited part of the operating range of the test bench, i.e. the speed is limited between approximately $150 \mathrm{rad} / \mathrm{s}$ and $250 \mathrm{rad} / \mathrm{s}$ and the maximum torque is $250 \mathrm{~N} \mathrm{~m}$

The closed-loop consisting of the proposed multivariable controller and the test bench is shown in Fig. 7, where the integral behavior of the speed dynamics is highlighted. The controller is given by the transfer functions $K_{i, j}, i, j=1,2$, while the plant description comes from (4). As already shown in Section 2, the variables of interest are the engine speed $\omega_{E}$ and the shaft torque $T_{S T}$, the controller sets the accelerator pedal position $\alpha$ and the set value $T_{D, s e t}$ of the dynamometer torque.

The order $n=1$ of the controller is chosen, hence the controller subsequently used to perform measurements at the test bench is a multivariable PI controller. The desired performance for the speed loop and the torque loop is given in terms of the settling time. The reference model used to design the multivariable PI controller is then the discretization of

$\mathbf{M}(s)=\left[\begin{array}{cc}\frac{1}{\tau_{1} s+1} & 0 \\ 0 & \frac{1}{\tau_{2} s+1}\end{array}\right]$,

where $\tau_{1}=(1 / 2 \pi) \frac{1}{2.5} s, \quad \tau_{2}=(1 / 2 \pi) \frac{1}{0.7} s$ and $s$ being the Laplace variable.

In the following, the performance of the proposed strategy is illustrated and compared to the industrial state-of-the-art controller using two different tests, illustrated in Figs. 8 and 9. In the figures, the first graphs show the accelerator pedal position $\alpha$, the second graphs the set value $T_{D \text {,set }}$ of the dynamometer torque $T_{D}$. The third graph and the fifth graph show the outputs of the test bench - the shaft torque $T_{S T}$ and the engine speed $\omega_{E}$ - with their references. The fourth graph shows the tracking error between the desired shaft torque $T_{S T, r e f}$ and the shaft torque $T_{S T}$, the sixth graph
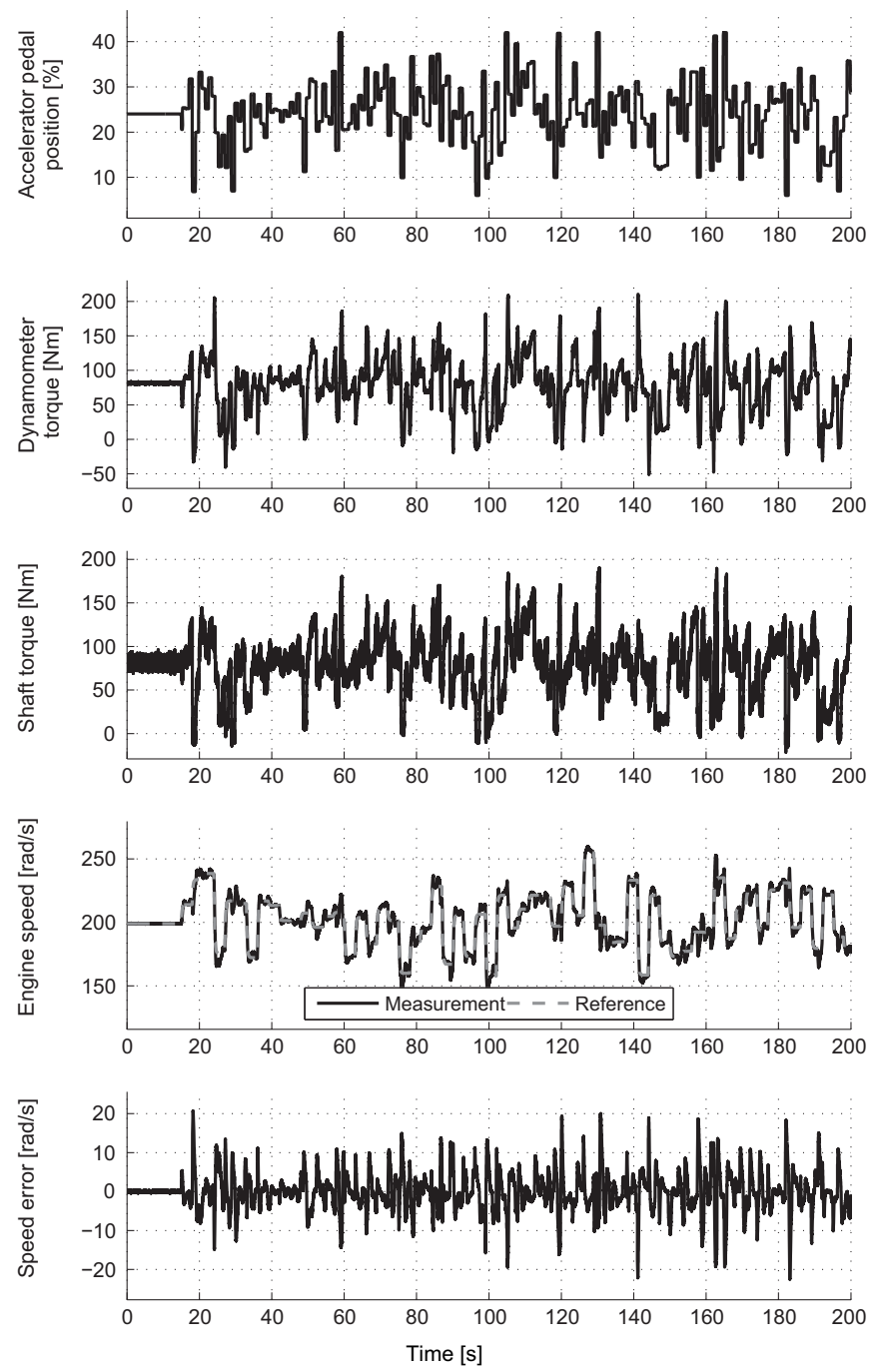

Fig. 6. Data-set consisting of accelerator pedal position $\alpha$ (input data; first graph), dynamometer torque $T_{D}$ and shaft torque $T_{S T}$ (output data; second graph and third graph, respectively) and desired engine speed $\omega_{E, \text { ref }}$ as well as measured engine speed $\omega_{E}$ (reference and output data, respectively; fourth graph) used to identify the parameters of the controller. The fifth graph shows the tracking error between the desired engine speed $\omega_{E \text {,ref }}$ and the measured engine speed $\omega_{E}$.

the tracking error between the desired engine speed $\omega_{E, \text { ref }}$ and the engine speed $\omega_{E}$. Note that in control of internal combustion engine test benches the mean torque and the mean rotational speed need to be tracked and that therefore the measurements are filtered filtering is done with an acausal filter to avoid a phase shift - for calculating and plotting of the tracking error. Ramped changes of the two references have been made in the first experiment, see Fig. 8. Fig. 9 instead shows the simulation of real measurements recorded in a vehicle at the test bench. For this purpose, the engine speed $\omega_{E}$ is read from the Controller Area Network (CAN) bus and the torque at the power train is measured using strain gauges during a test drive. Subsequently, these measure-ments in a vehicle are used as references for the second experiment at the test bench.

\section{Discussion}

In this section, the performance of the proposed approach is compared to the industrial standard. The differences between these two controllers should not be disregarded. The industrial 


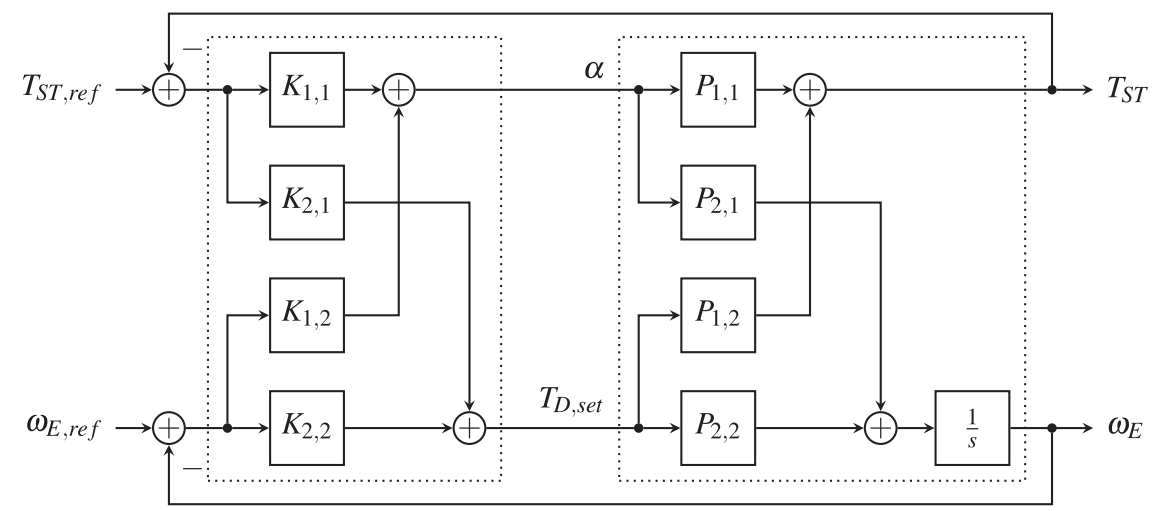

Fig. 7. Structure of the closed-loop consisting of the internal combustion engine test bench and the proposed multivariable controller.
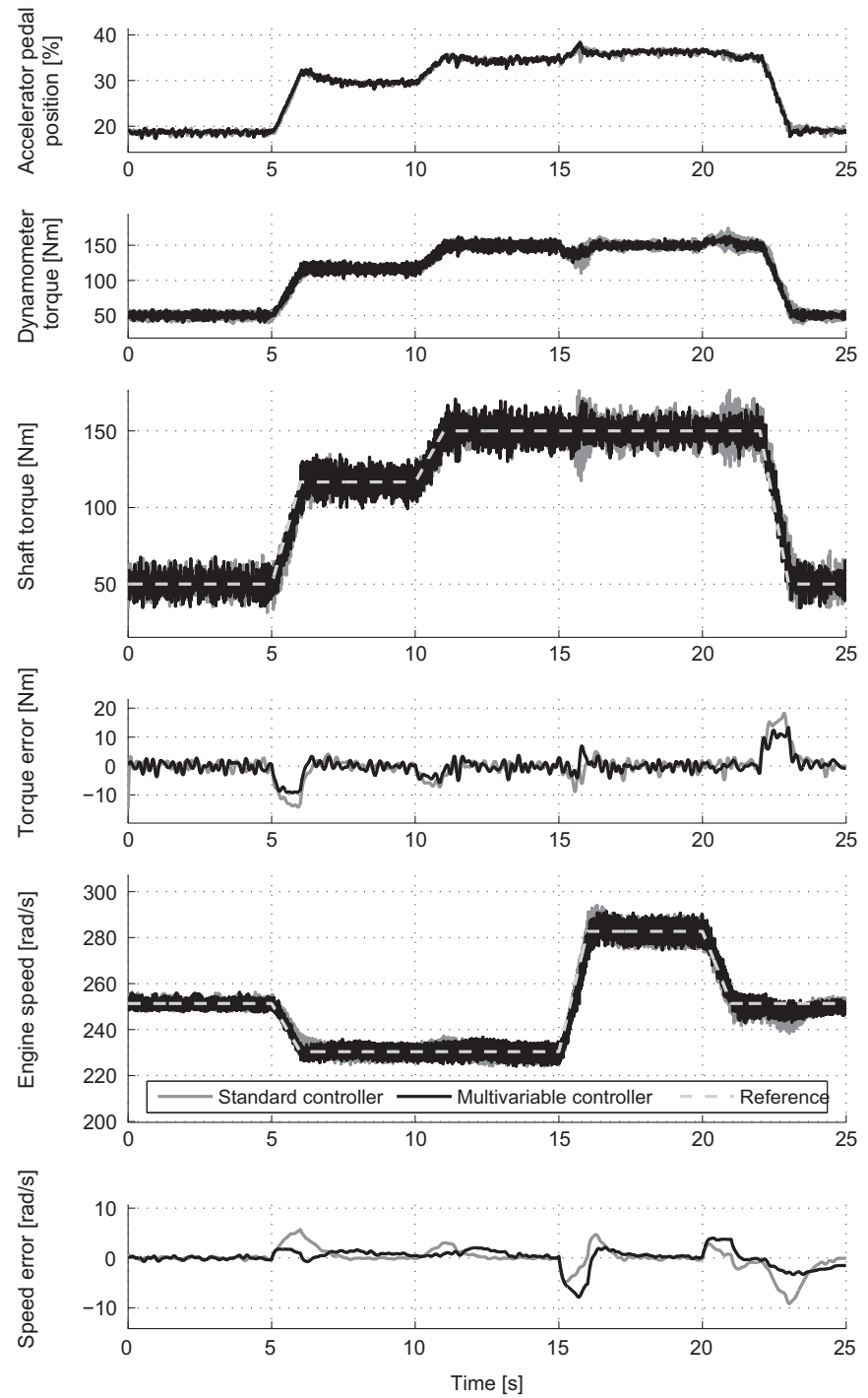

Fig. 8. Comparison of tracking of shaft torque $T_{S T}$ (third graph) and engine speed $\omega_{E}$ (fifth graph) for a simple experiment using the industrial standard controller and the proposed multivariable controller. The fourth graph and the sixth graph show the tracking error between the desired shaft torque $T_{S T \text { ref }}$ and the shaft torque $T_{S T}$ as well as the tracking error between the desired engine speed $\omega_{E, \text { ref }}$ and the engine speed $\omega_{E}$.

standard consists of two SISO control loops that are often tuned heuristically, the controller parameters are adjusted depending on the operating point. The parameters of the proposed, multivariable controller are directly identified from measurements.
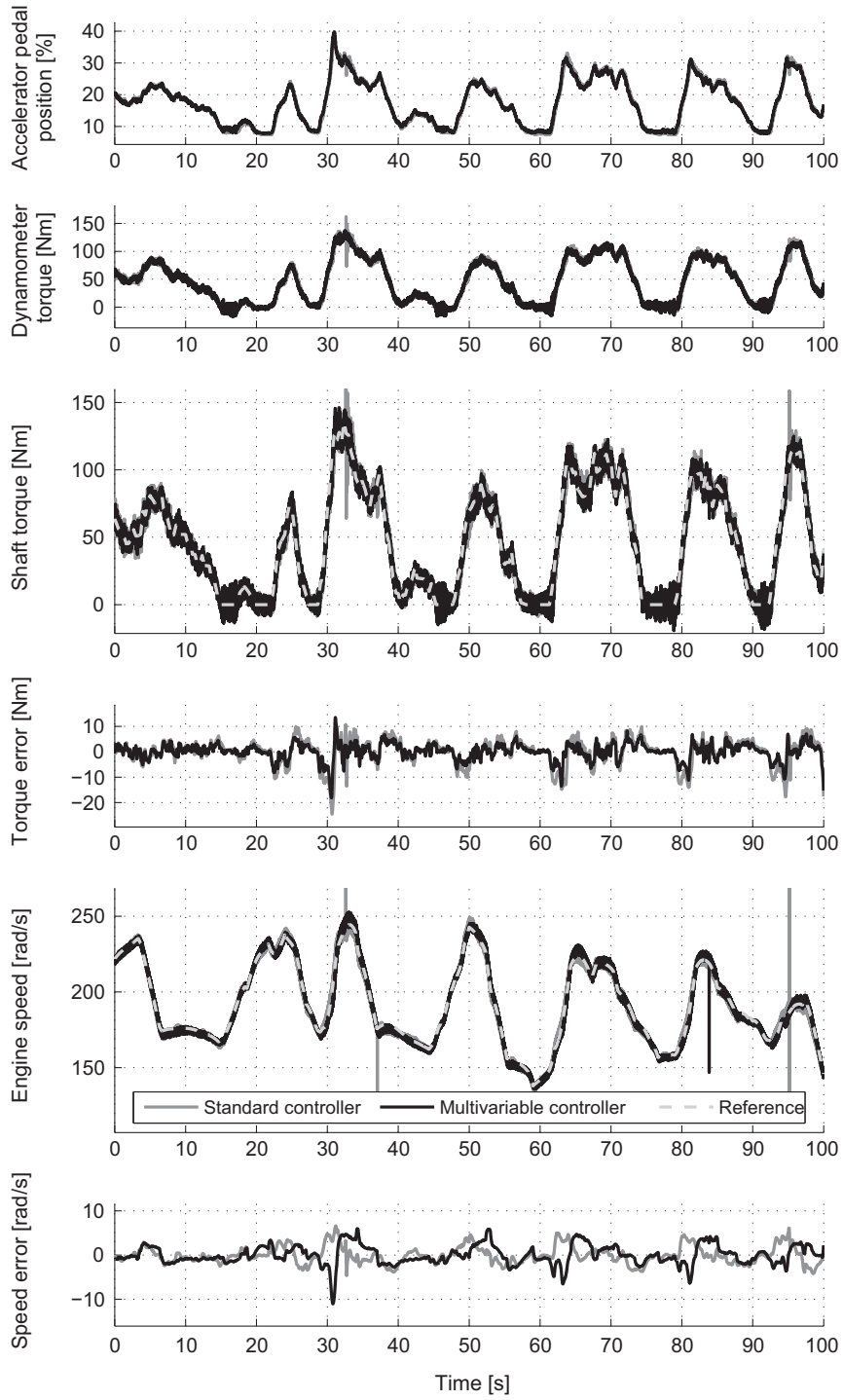

Fig. 9. Comparison of tracking of shaft torque $T_{S T}$ (third graph) and engine speed $\omega_{E}$ (fifth graph) for the simulation of measurements (recorded in a vehicle) at a test bench using the industrial standard controller and the proposed multivariable controller. The fourth graph and the sixth graph show the tracking error between the desired shaft torque $T_{S T \text { ref }}$ and the shaft torque $T_{S T}$ as well as the tracking error between the desired engine speed $\omega_{E \text {,ref }}$ and the engine speed $\omega_{E}$. The spikes in all graphs result from the error in the speed measurement.

By looking at Fig. 8, it is evident that the tracking performance is good for both controllers, however, significant improvements can be highlighted in the rejection of the coupling effects. For 
example, the disturbance of the shaft torque $T_{S T}$ is significantly lowered when increasing the engine speed $\omega_{E}$ at $t=15 \mathrm{~s}$. The same applies for changes in the shaft torque $T_{S T}$, see Fig. 8 at $t=22 \mathrm{~s}$. This is due to the fact that, in internal combustion engine test benches, the dynamometer torque is usually built up faster than the torque of the internal combustion engine. In a multivariable controller, such an actuator can be used for both output channels and not only for the engine speed $\omega_{E}$. This is evident by looking at the input trajectories in Fig. 8. Moreover, the two control variables can compensate each other by taking into account the tracking errors of both the controlled outputs, see Fig. 10.

Note that the single multivariable controller can be found by simply performing an experiment that explores the entire operating range of the test bench. The tracking performance of this controller is shown in Figs. 8 and 9. An adaptation of the controller parameters depending on the operating point or nonlinear static compensation maps can be avoided. However, note that this property cannot be guaranteed for other applications.

The parametrization of the controller (6) enforces an integral action in the controller. Note that the speed loop already contains an integrator that is canceled by the zero of the resulting proportional-integral-controller in $K_{2,2}$. The action of the accelerator pedal position $\alpha$ on the engine speed $\omega_{E}$ is usually slower than the action of the dynamometer torque $T_{D \text {,set }}$. Thus, the compensation by $K_{2,1}$ - acting on a system described by the input sensitivity function from the output of $K_{2,1}$ to the engine speed $\omega_{E}-$ is slower than the compensation by $K_{2,2}$ as well. Therefore, $K_{2,1}$ acts on the stabilized speed control loop and the integral action of $K_{2,1}$ is automatically kept to guarantee for zero steady-state error.

The performance of the two controllers is comparable also for what concerns the tests in Fig. 9, with the only difference that, being the test less dynamically challenging, the decoupling action is acceptable in both cases. Nevertheless, the design phase for the data-driven controller requires only a few seconds, while a modelbased controller usually takes time and experimental effort to adjust the controller parameters to compensate for modeling errors. This fact may be a great advantage of the data-driven
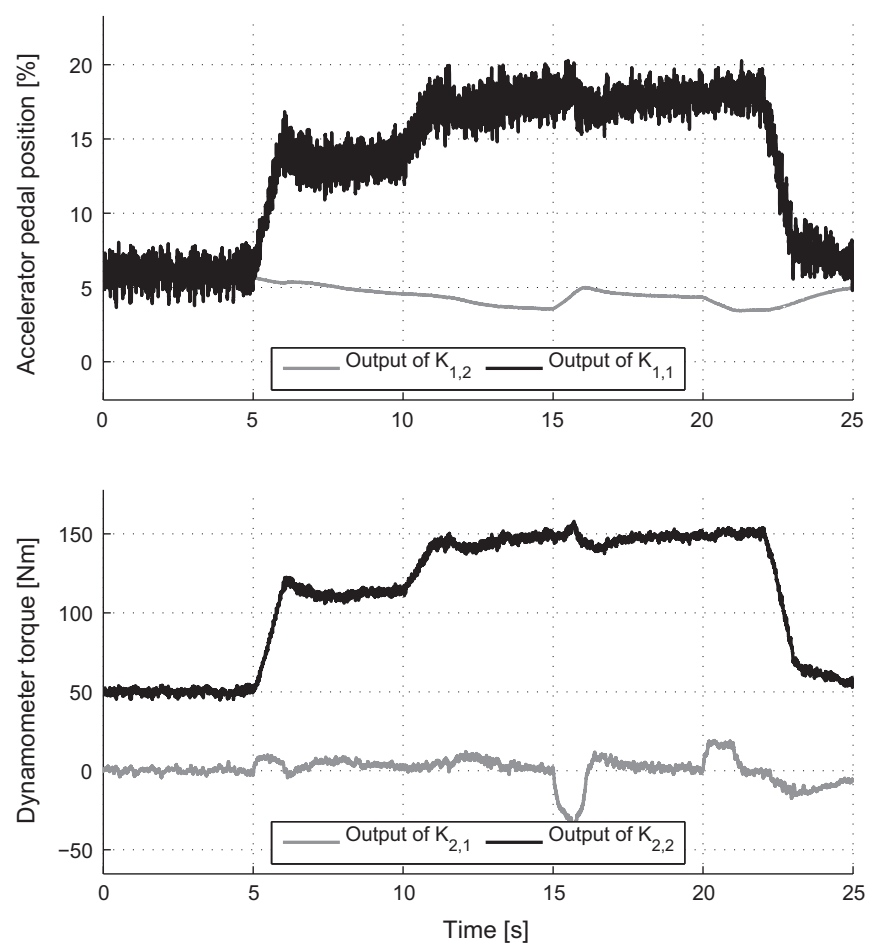

Fig. 10. Inputs to the test bench for a simple experiment are divided into control signals for tracking (dark) and control signals for decoupling (light).
Table 1

Comparison of Root Mean Square Error (RMSE) of shaft torque $T_{S T}$ and engine speed $\omega_{E}$ using both the standard controller and the proposed controller for the experiments shown in Figs. 8 and 9, respectively.

\begin{tabular}{|c|c|c|c|c|}
\hline \multirow[t]{2}{*}{ Controller } & \multicolumn{2}{|c|}{ Simple experiment } & \multicolumn{2}{|c|}{ Simulation of measurements } \\
\hline & $\begin{array}{l}\text { Torque } \\
(\mathrm{N} \mathrm{m})\end{array}$ & $\begin{array}{l}\text { Speed } \\
(\mathrm{rad} / \mathrm{s})\end{array}$ & $\begin{array}{l}\text { Torque } \\
(\mathrm{N} \mathrm{m})\end{array}$ & $\begin{array}{l}\text { Speed } \\
(\mathrm{rad} / \mathrm{s})\end{array}$ \\
\hline Standard controller & 4.13 & 2.16 & 4.97 & 2.04 \\
\hline Proposed controller & 3.06 & 1.8 & 3.21 & 2.16 \\
\hline
\end{tabular}

approach in case of hardware changes or system degradation, which would still require controller re-tuning, but on a faster way.

Table 1 shows a comparison of the Root Mean Square Error (RMSE) for both the standard controller and the proposed controller for the experiments shown in Figs. 8 and 9, respectively.

Note that the engine torque $T_{E}$ is generated from the sum of the torque of all cylinders. The combustion cycle can be divided into four phases for each cylinder. Each cylinder produces a positive torque only in the phase in which the combustion takes place and accelerates the piston. The torque of a cylinder is negative in the three other phases. These oscillations of the engine torque $T_{E}$ translate to the shaft torque $T_{S T}$ and the engine speed $\omega_{E}$ and can be seen in Figs. 8 and 9. Note that in the control of internal combustion engine test benches it is not crucial to compensate all these oscillations, which are outside the interesting frequency range, but rather to make the mean torque track a desired value.

\section{Conclusion and outlook}

In this paper, the direct data-driven control system design for an internal combustion engine test bench is proposed and discussed. The method is mainly based on Formentin, Savaresi, et al. (2012), but an extension is introduced to guarantee consistency also when using closed-loop data.

The resulting controller applied on the test bench system provides a similar performance in tracking the desired engine speed and the shaft torque to that of the industrial state-of-the-art controller. However, three advantages of the proposed method arise:

- since the coupling terms are taken into account in the MIMO structure, they can be better compensated compared to simple SISO control loops (see e.g. the tracking error shown in the fourth graph and sixth graph of Fig. 8 and the input signals shown in Fig. 10);

- no model of the test bench is required to tune the controller parameters;

- nonlinear maps used in the state-of-the-art controllers to adapt to different operating conditions are not required even though analogous performance is given.

Obviously, due to the nonlinearity of the plant, all interesting areas of the operating range - engine speed and shaft torque - have to be visited during the identification experiment to provide a good average performance.

Future works will focus on the development of a data-driven technique taking explicitly into account the nonlinear nature of the system.

\section{Acknowledgements}

The authors gratefully acknowledge the sponsoring of this work by the COMET K2 Center "Austrian Center of Competence 
Table A1

List of system inputs and outputs.

\begin{tabular}{lll}
\hline Variable & Unit & Description \\
\hline$\alpha$ & $\%$ & Accelerator pedal position \\
$T_{D}$ & $\mathrm{~N} \mathrm{~m}$ & Dynamometer torque \\
$T_{D, \text { set }}$ & $\mathrm{N} \mathrm{m}$ & Set value of dynamometer torque \\
$T_{E}$ & $\mathrm{~N} \mathrm{~m}$ & Torque generated by the internal combustion engine \\
$T_{S T}$ & $\mathrm{~N} \mathrm{~m}$ & Shaft torque \\
$T_{S T, r e f}$ & $\mathrm{~N} \mathrm{~m}$ & Desired shaft torque \\
$\omega_{D}$ & $\mathrm{rad} / \mathrm{s}$ & Dynamometer speed \\
$\omega_{E}$ & $\mathrm{rad} / \mathrm{s}$ & Engine speed \\
$\omega_{E, \text { ref }}$ & $\mathrm{rad} / \mathrm{s}$ & Desired engine speed \\
\hline
\end{tabular}

Table A2

List of system parameters.

\begin{tabular}{|c|c|c|}
\hline Variable & Unit & Description \\
\hline$c$ & $\mathrm{~kg} \mathrm{~m}^{2} / \mathrm{s}^{2}$ & Stiffness of connecting shaft \\
\hline$d$ & $\mathrm{~kg} \mathrm{~m}^{2} / \mathrm{s}$ & Damping of connecting shaft \\
\hline$\Theta_{D}$ & $\mathrm{~kg} \mathrm{~m}^{2}$ & Inertia of dynamometer including the proportionale inertia of the connecting shaft and the adapter flange \\
\hline$\Theta_{E}$ & $\mathrm{~kg} \mathrm{~m}^{2}$ & Inertia of internal combustion engine including the proportionale inertia of the connecting shaft and the adapter flange \\
\hline$m(\cdot)$ & $\mathrm{N} \mathrm{m}$ & Stationary engine torque of the internal combustion engine \\
\hline$\tau(\cdot)$ & $\mathrm{s}^{-1}$ & Time constant of internal combustion engine \\
\hline
\end{tabular}

in Mechatronics (ACCM)". The COMET Program is funded by the Austrian federal government, the Federal State Upper Austria and the Scientific Partners of ACCM.

\section{Appendix A. List of variables}

In Tables A1 and A2 the inputs and outputs of the system and the system parameters are summarized, respectively.

\section{References}

Bazanella, A. S. (2012). Data-driven controller design: The H2 approach, communications and control engineering. Springer Science+Business Media.

Bunker, B. J., Franchek, M. A., \& Thomason, B. E. (1997). Robust multivariable control of an engine-dynamometer system. IEEE Transactions on Control Systems Technology, 5(2), 189-199, http://dx.doi.org/10.1109/87.556024.

Campi, M. C., Lecchini, A., \& Savaresi, S. M. (2002). Virtual reference feedback tuning: A direct method for the design of feedback controllers. Automatica, 38(8), 1337-1346 http://dx.doi.org/10.1016/S0005-1098(02)00032-8.

Formentin, S., Hirsch, M., Savaresi, S. M., \& del Re, L. (2012). Direct data-driven control of a Diesel engine airpath. In 2012 American control conference (ACC) (pp. 4198-4203), Montreal, QC, Canada.

Formentin, S., \& Savaresi, S. M. (2011a). Noniterative data-driven design of multivariable controllers. In 2011 50th IEEE conference on decision and control and European control conference (CDC-ECC) (pp. 5106-5111), Orlando, FL, USA. http://dx.doi.org/10.1109/CDC.2011.6160388.

Formentin, S., \& Savaresi, S. M. (2011b). Virtual reference feedback tuning for linear parameter-varying systems. In 18th IFAC world congress (pp. 10219-

10224), Milano, Italy. http://dx.doi.org/10.3182/20110828-6-IT-1002.01184.

Formentin, S., Savaresi, S. M., \& del Re, L. (2012). Noniterative direct data-driven tuning of multivariable controllers: theory and application. IEE Proceedings -Control Theory and Applications, 6(9), 1250-1257, http://dx.doi.org/10.1049/ iet-cta.2011.0204.

Gruenbacher, E., Colaneri, P., \& del Re, L. (2008). Guaranteed robustness bounds for matched-disturbance nonlinear systems. Automatica, 44(9), 2230-2240, http://dx. doi.org/10.1016/j.automatica.2007.12.018.
Gruenbacher, E. \& del Re, L. (2008). Robust inverse control for combustion engine test benches. In 2008 American control conference (ACC) (pp. 2852-2857) Seattle, WA, USA. http://dx.doi.org/10.1109/ACC.2008.4586926.

Hjalmarsson, H., Gevers, M., Gunnarsson, S., \& Lequin, O. (1998). Iterative feedback tuning: Theory and applications. IEEE Control Systems Magazine, 18(4), 26-41, http://dx.doi.org/10.1109/37.710876.

Ljung, L. (1998). System identification: Theory for the user (2nd ed.). Prentice Hall.

Ortner, P., Gruenbacher, E., \& del Re, L. (2008). Model based nonlinear observers for torque estimation on a combustion engine test bench. In 2008 IEEE conference on control applications (CCA) (pp. 221-226), Hagen, Germany. http://dx.doi.org/ 10.1109/CCA.2008.4629628.

Passenbrunner, T., Formentin, S., Savaresi, S., \& del Re, L. (2012). Direct data-driven control of internal combustion engine test benches using closed-loop experiments. In: 2012 51st annual IEEE conference on decision and control (CDC)(pp. 37653770), Maui, HI, USA. http://dx.doi.org/10.1109/CDC.2012.6425955.

Passenbrunner, T., Sassano, M., \& del Re, L. (2011). Approximate optimal control of internal combustion engine test benches. In 2011 50th IEEE conference on decision and control and European control conference (CDC-ECC) (pp. 8050-8055), Orlando, FL, USA, 2011. http://dx.doi.org/10.1109/CDC.2011.6161120.

Passenbrunner, T., Trogmann, H., \& del Re, L. (2012). Unknown input high-gain observer for internal combustion engine test benches. In 2012 American control conference (ACC) (pp. 6763-6768), Montreal, QC, Canada.

PUMA Open - avl.com, 〈https://www.avl.com/puma-open〉, (accessed: 2014-02-28).

Soderstrom, T., \& Stoica, P. G. (1983). Instrumental variable methods for system identification. Lecture notes in control and information sciences. New York: Springer-Verlag.

Stoica, P. G., \& Jansson, M. (2002). MIMO system identification: State-space and subspace approximations versus transfer function and instrumental variables. IEEE Transactions on Signal Processing, 48(11), 3087-3099, http:// dx.doi.org/10.1109/78.875466.

Tuken, T., Fullmer, R. R., \& VanGerpen, J. (1990). Modeling, identification, and torque control of a Diesel engine for transient test cycles. In SAE international congress \& exposition (pp. 39-49), Detroit, MI, USA, paper 900235. http://dx.doi.org/10. 4271/900235.

Wellstead, P. E., \& Zarrop, M. B. (1991). Self-tuning systems: Control and signal processing. John Wiley \& Sons, Inc.

Yanakiev, D. (1998). Adaptive control of Diesel engine-dynamometer systems.

In 37th IEEE conference on decision and control (CDC) (Vol. 2, pp. 1530-1534), Tampa, FL, USA. http://dx.doi.org/10.1109/CDC.1998.758506.

Yeddanapudi, M., \& Potvin, A. F. (1997). Nonlinear control design blockset:

Usergride. Natick, MA: The Mathworks Inc. 